Ani. Génét. Sél. anim., I979, 11 (3), 25I-266.

\title{
Déterminisme génétique \\ de la ponte de femelles vierges de Tribolium castaneum en milieu standard et en milieu dégradé $\left({ }^{1}\right)$
}

\author{
G. BOLET, Araceli GALLEGO* et F. OROZCO** \\ Station de Génétique quantitative et appliquée, \\ Centre national de Recherches zootechniques, I.N.R.A., \\ 78350 Jouy-en-Josas, France \\ * Departamento de Genética, Facultad de Biologia, \\ Universidad Complutense de Madrid, Ciudad Universitaria, \\ Madrid 3, Espagne \\ **Departamento de Genética, Instituto Nacional de Investigaciones Agrarias, \\ Carretera de la Coruña, Madrid 35, Espagne
}

\begin{abstract}
Résumé
Pour étudier le déterminisme génétique de la résistance à des conditions de milieu défavorables, et notamment tester l'hypothèse qu'elle est due essentiellement à l'action de gènes non additifs, nous avons utilisé le caractère "ponte de femelles vierges de Tribolium castaneum du $7^{\mathrm{e}}$ au I I $^{\mathbf{e}}$ jour (première période) et du I $^{\mathbf{e}}$ au $\mathrm{I}_{5}^{\mathrm{e}}$ jour (deuxième période) de la vie adulte, les animaux étant placés dans le milieu d'élevage standard (S) ou dans un milieu fortement dégradé (D). Dans une première expérience, nous avons créé trois groupes constitué chacun de 600 femelles puis de 3 filles de chacune dans la succession de milieux suivante, respectivement S.S., S.D., D.D. (première et deuxième période). La ponte en milieu dégradé est inférieure de 60 p. roo à celle en milieu standard. L'héritabilité n'est pas modifiée significativement par la période ou le milieu de ponte. Par contre, quelle que soit la période ou le milieu, l'héritabilité calculée par corrélation entre les 3 pleines sœurs $(0,33)$ est significativement supérieure à celle calculée par régression des filles sur la mère $(0,22)$ ce qui peut être dû à des effets génétiques non additifs et /ou un effet milieu commun chez les filles. Dans une deuxième expérience, nous avons sélectionné les animaux à l'aide d'un indice avec restriction permettant d'augmenter la ponte en milieu dégradé $\left(\mathbf{P}_{d}\right)$ en deuxième période sans modifier celle en milieu standard $\left(\mathrm{P}_{8}\right)$ en première période. $\mathrm{Si}$, comme prévu, la sélection familiale n'a eu d'effet ni sur $\mathrm{P}_{\delta}$, ni sur $\mathrm{P}_{d}$, la sélection réciproque récurrente, contrairement à nos hypothèses, n'a eu aucun effet sur $\mathrm{P}_{\boldsymbol{d}}$ et a fait augmenter $\mathrm{P}_{\boldsymbol{s}}$. I,es deux principales conclusions de ces expériences sont, d'une part que les gènes à effet additif sont identiques en milieu standard ou dégradé, d'autre part qu'il est impossible de conclure catégoriquement à l'existence de gènes de résistance agissant par dominance.
\end{abstract}

(1) I es travaux décrits dans cet article ont été réalisés au Iaboratoire đu Département de génétique de 1'I.N.I.A., à Madrid. 


\section{Introduction}

Le problème du déterminisme génétique de la résistance des animaux à des conditions de milieu défavorables intéresse de plus en plus les chercheurs en productions animales; il est vrai qu'il trouve son application dans de nombreux domaines, comme l'utilisation de l'élevage pour la mise en valeur de milieux défavorisés, et constitue un élément de réponse au vaste problème des interactions entre génotype et milieu (critères de sélection, choix du milieu de sélection des animaux doniestiques...).

Les expériences à ce sujet sont, à l'heure actuelle, assez peu nombreuses même sur animaux de laboratoire. BARNETT et COLEMAN (I960), à partir d'expériences sur Souris, et PARSONS (I97I), dans the revue des travaux sur Drosophile montrent que les animaux résistent d'autant mieux à un milieu défavorable que leur degré d'hétérozygotie est élevé.

Sur Tribolium Castaneum, petit coléoptère de la famille des Ténébrionidés qui présente un cycle de développement sans diapause de 30 jours dans les conditions de lakoratoire, la rapidité du cycle, la possibilité d'identifier chaque animal et de mesurer individuellement ses performances permettent d'appliquer des schémas d'accouplement et de sélection très bien adaptés à l'étude de ce thème. C'est ainsi que plusieurs auteurs ont utiliséle caractère " ponte de femelles vierges du septième au onzième jour de la vie adulte ": pour OrOzco et BELL, (I974a et $b$ ) et Orozco (I976), étudiant l'effet de la température ambiante sur la ponte, l'expression d'un caractère quantitatif pourrait être régie par deux types de gènes : des gènes directement responsables de l'expression du caractère et agissant essentiellement de façon additive, et des gènes contrôlant par surdominance et/ou d'autres effets non additifs la résistance générale à tout type de situation adverse. Par cortre, LOPEZ-FANJUL, et JODAR (I977) montrent que ce caractère de ponte a un déterminisme essentiellement additif, que la température soit normale $\left(33^{\circ} \mathrm{C}\right)$ ou défavorable $\left(28^{\circ} \mathrm{C}\right.$ ). Selon Lavie et al. (I978), pour ce même caractère de ponte, la résistance au stress (un milieu d'élevage dégradé) serait contrólée par un faible nombre de loci, mais ces auteurs ne peuvent se prononcer sur le mode d'action des gènes présents à ces loci.

Dans la présente étude nous nous sommes efrorcés, en utilisant le même caraztère de ponte de mettre en évidence le déterminisme génétique de la résistance à un milieu adverse de deux façons : d'une part par l'étude des paramètres génétiques de la ponte en milieu normal et en rilieu défavorable (expérience I), d'autre part en sélectionnant la ponte en rrilieu défavorable en essayant d'agir uniquement sur ces éventtels gènes de résistance, non additifs, et non sur ceux, additifs, contrôlant le caractère en milieu optimal (expérience II).

\section{Matériel et méthodes}

\section{1. - Matériel animal. Milieux utilisés}

Le caractère étudié est la ponte d'œufs de la femelle vierge de Tribolium Castaneum du $7^{\mathrm{e}}$ au I $\mathrm{I}^{\mathrm{e}}$ jour, puis du $\mathrm{II}^{\mathrm{e}}$ au $\mathrm{I}^{\mathrm{e}}$ jour de la vie adulte, à une température de $33^{\circ} \mathrm{C} \pm 0,5^{\circ} \mathrm{C}$ et une humidité relative de $70 \mathrm{p}$. IOO $\pm 3 \mathrm{p}$. Ioo dans deux milicux de ponte différents : 
- le milieu standard composé de $95 \mathrm{p}$. Ioo de farine de blé et $5 \mathrm{p}$. roo de levure de bière, finement criblées et mélangées;

- le milieu dégradé, obtenu à partir du milieu standard dans lequel on a élevé une population de ces insectes pendant un mois à une densité initiale de 30 individus par gramme de milieu; le milieu, ayant perdu une grande partie de sa valeur alimentaire et s'étant chargé de produits de déjections et de dérivés de quinones, prend une couleur brun clair; avant utilisation, il est passé à l'étuve et criblé finement.

La totalité du milieu nécessaire a été préparée en une seule fois. La ponte des femelles est obtenue individuellement dans un tube de verre contenant deux grammes de milieu standard ou dégradé, puis recueillie par criblage.

\section{2. - Protocole expérimental}

L'expérience s'est déroulée en deux parties successives et indépendantes, l'une de type statique, l'autre de type dynamique.

\section{a) Expérience de type statique (expérience $I$ )}

A partir de la descendance de $I 67$ couples réalisés au hasard, trois groupes (S-S, S-D, D-D) ont été formés, chacun composé d'un descendant mâle et d'un descendant femelle de chacun des couples; dans chaque groupe, on a mesuré la ponte des femelles vierges en période I puis 2 , et on les a accouplées au hasard à un mâle afin de donner chacune trois filles dont la ponte en période I puis 2 était également mesurée. La différence entre les 3 groupes était le milieu de ponte pour chacune des deux périodes (fig. I, tabl. I); les caractères mesurés chez la mère et chez ses trois filles sont ainsi les mêmes (même milieu de ponte pour la même période). Quatre répétitions identiques de cette unité ont été réalisées successivement.

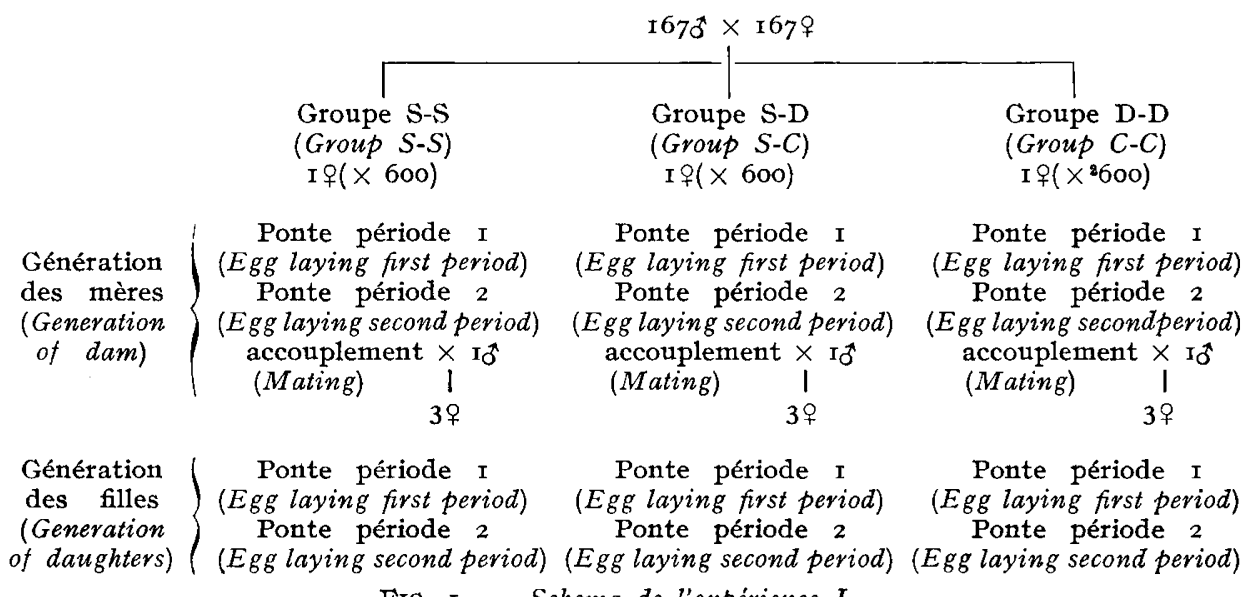

FIG. I. - Schema de l'expérience $I$.

Scheme of experiment $I$. 
TABLEAU I

Milieux de ponte pour les trois groupes de l'expérience I Egg laying environment for the three groups of the experiment $I$

\begin{tabular}{|c|c|c|c|}
\hline Période $($ Proupe (Group) & $\begin{array}{l}\text { S-S } \\
(S-S)\end{array}$ & $\begin{array}{l}\mathrm{S}-\mathrm{D} \\
(S-C)\end{array}$ & $\begin{array}{l}\mathrm{D}-\mathrm{I}) \\
(C-C)\end{array}$ \\
\hline 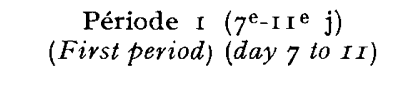 & $\begin{array}{l}\text { Milieu standard } \\
\text { (Standard medium) }\end{array}$ & $\begin{array}{l}\text { Milieu standard } \\
\text { (Standard medium) }\end{array}$ & $\begin{array}{l}\text { Milieu dégradé } \\
\text { (Conditioned } \\
\text { medium) }\end{array}$ \\
\hline $\begin{array}{l}\text { Période } 2\left(\mathrm{II}^{\mathrm{C}}-\mathrm{I} 5^{\mathrm{e}} \mathrm{j}\right) \\
\text { (Second period) (day II to I } 5)\end{array}$ & $\begin{array}{l}\text { Milieu standard } \\
\text { (Standard medium) }\end{array}$ & $\begin{array}{l}\text { Milieu dégradé } \\
\text { (Conditioned } \\
\text { medium })\end{array}$ & $\begin{array}{l}\text { Milieu dégradé } \\
\text { (Conditioned } \\
\text { medium) }\end{array}$ \\
\hline
\end{tabular}

\section{b) Expérience de type dynamique (expérience $I I$ )}

Les femelles vierges, dans cette deuxième expérience, subissent un traitement analogue à celles du groupe S-D de l'expérience I, c'est-à-dire que l'on mesure individuellement leur ponte pour période I en milieu standard, puis pour la période 2 en milieu dégradé.

Pour créer trois populations $(\mathrm{A}, \mathrm{B}, \mathrm{C})$ de 40 à 45 familles chacune par génération, nous avons réalisé $\mathrm{I} 35$ accouplements individualisés au hasard, au sein de l'ensemble de la population Consejo. 5 descendants mâles et 5 à ro descendants femelles de chaque couple constituent une famille. Nous avons utilisé ces 3 populations pour réaliser deux types de sélection :

- avec les populations $\mathrm{A}$ et $\mathrm{B}$, une sélection réciproque récurrente : à chaque génération, 9 mâles $A$ et 9 mâles $B$ sont sélectionnés sur les performances de leurs cinq filles croisées, $\mathrm{A} \times \mathrm{B}$ et $\mathrm{B} \times \mathrm{A}$ respectivement; ils sont accouplés, en évitant les couples frère $X$ sœur, chacun avec une femelle prise au hasard dans leur population et donnent chacun des descendants mâles et des descendants femelles qui constituent les deux populations de la génération suivante (fig. 2);

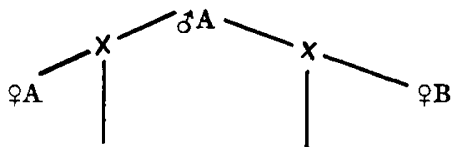

Reproduction des mâles sélectionnés (Reproduction of selected sires)
$5 \%(A \times B)$<smiles>C=CC</smiles>

Sélection des pères

(Selection of sires)
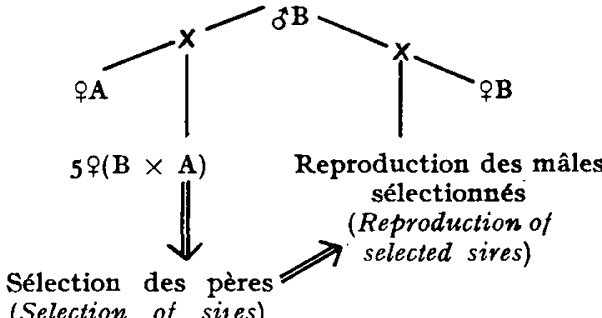

FIG. 2. - Sélection réciproque récurrente de l'expérience II. Reciprocal recurrent selection of the experience $I I$.

- avec la population $C$, une sélection familiale : sur les 45 familles de 5 pleines sœurs mises en contrôle, 9 sont sélectionnées ainsi que leurs pleins frères. Les 45 mâles et femelles sont accouplés individuellement en évitant les couples frères $\times$ sœurs pour donner la génération suivante. 
Critère de sélection : Les individus sont sélectionnés sur un indice avec restriction (KEMPTHORNE et NORDSKOG, I959) calculé afin de réaliser un progrès génétique sur la ponte en milieu dégradé en maintenant constante la ponte en milieu standard. vante :

L'expression générale de l'indice pour les deux types de sélection est la sui-

$$
\mathrm{I}=\mathrm{P}_{d}-b \mathrm{P}_{\mathrm{S}}
$$

où $\mathrm{P}_{\boldsymbol{d}}$ représente la ponte de la femelle vierge en milieu dégradé $\left(7^{\mathrm{e}}-\mathrm{II}{ }^{\mathrm{e}}\right.$ jour$)$,

$\mathrm{P}_{\mathrm{S}}$ représente la ponte de la femelle vierge en milieu standard (I I ${ }^{\mathrm{e}}-\mathrm{I}_{5} \mathrm{e}^{\mathrm{e}}$ jour),

$b$ est la régression génétique de la ponte en milieu dégradé sur la ponte en milieu standard.

Le coefficient $b$ utilisé pour la première génération a été calculé à partir des données du groupe S-D de l'expérience I; puis pour chaque génération, il a été recalculé à partir de l'ensemble des données disponibles (expérience $I+$ générations antérieures).

Pour les deux types de sélection, le critère de sélection est l'indice moyen des cinq pleines sœurs mises en contrôle; s'il en reste moins de 3 à la fin du contrôle dans une famille l'indice de celle-ci n'est pas calculé. Deux répétitions identiques à une semaine d'intervalle de ce schéma ont été réalisées.

\section{3. - Analyses statistiques}

\section{a) Expérience $I$}

Pour chacune des 6 cellules période de ponte $\times$ milieu de ponte (voir tabl. I), pour chaque répétition nous avons calculé les moyennes et variances pour les deux générations et la régression linéaire de la moyenne de ponte des filles sur la ponte de la mère.

Cette analyse permet de calculer l'héritabilité $\left(h_{\mathrm{R}}^{2}\right)$ d'un caractère à partir $\mathrm{du}$ coefficient de régression $\beta: h_{\mathrm{n}}^{2}=2 \beta$.

L'analyse de variance et de covariance des données de la génération des filles est partie du modèle suivant :

$$
\mathrm{X}_{i j}=\mu+\mathrm{F}_{i}+\mathrm{E}_{i j}
$$

$\mathrm{X}_{i j}$ : valeur pour la $j^{i \text { eme }}$ femelle de la $i$ ieme famille du caractère mesuré,

$\mu$ : moyenne générale,

$F_{i}$ : effet de la $i$ ieme famille; $F_{i}$ est une variable aléatoire de loi normale $\mathrm{N}\left(\mathrm{O}, \sigma_{\mathrm{F}}^{2}\right)$,

$\mathrm{E}_{i j}$ : erreur résiduelle; $\mathrm{E}_{i j}$ est une variable aléatoire de loi normale $\mathrm{N}\left(\mathrm{O}, \sigma_{\mathrm{E}}^{2}\right)$.

Ces analyses de variance et de covariance permettent de calculer l'héritabilité

$\left(h_{\mathrm{s}}^{2}\right)$ d'un caractère à partir de la corrélation $t$ entre sœurs $\left(h_{\mathrm{s}}^{2}=2 t=\frac{2 \sigma_{\mathrm{F}}^{2}}{\sigma_{\mathrm{F}}^{2}+\sigma_{\mathrm{E}}^{2}}\right)$, la corrélation génétique et phénotypique entre deux caractères.

\section{b) Expérience II}

\section{- Calcul du coefficient " $b$ " de l'index}

A chaque génération, la régression génétique de la ponte en milieu dégradé sur la ponte en milieu standard est calculée en utilisant les variances et covariances 
génétiques estimées avec le même modèle d'analyse de variance et covariance que celui décrit ci-dessus. " $b$ " est égal au rapport de la composante familiale de la covariance entre $\mathrm{P}_{d}$ et $\mathrm{P}_{s}$ sur la variance familiale de $\mathrm{P}_{s}$.

$$
b=\frac{\operatorname{Cov}_{\mathrm{F}}\left(\mathrm{P}_{d}, \mathrm{P}_{s}\right)}{\sigma_{\mathrm{F}}^{2}\left(\mathrm{P}_{\mathrm{S}}\right)}
$$

\section{- Bilan de la sélection}

En utilisant l'indice de sélection avec restriction que nous avons défini, on peut calculer le progrès génétique attendu pour la ponte en milieu standard $\mathrm{E}\left(\Delta \mathrm{G}_{\delta}\right)$ et en milieu dégradé $\mathrm{E}\left(\Delta \mathrm{G}_{d}\right)$ par génération :

$$
\mathrm{E}\left(\Delta \mathrm{G}_{s}\right)=\mathrm{o} \text { dans les deux systèmes de sélection }
$$

(I) $\quad \mathrm{E}\left(\Delta \mathrm{G}_{d}\right)_{\mathrm{C}}=\frac{i}{\sigma \overline{\mathrm{I}}} \frac{2 n+\mathrm{I}}{4^{n}} \sigma_{\mathrm{\sigma}_{d}}^{2}\left(\mathrm{I}-r_{\mathrm{a}}^{2}\right)$ en sélection familiale

(2) $\quad \mathrm{E}\left(\Delta \mathrm{G}_{d}\right)_{\mathrm{A} \cdot \mathrm{B}}=\frac{i}{4 \sigma \overline{\mathrm{I}}} \sigma_{\mathrm{G}_{d}}^{2}\left(\mathrm{I}-\gamma_{\mathrm{a}}^{2}\right)$ en sélection réciproque récurrente

où $n=$ nombre d'individus mesurés,

$r_{t}=$ corrélation génétique entre $\mathrm{P}_{s}$ et $\mathrm{P}_{d}$,

$\sigma_{\mathrm{a}_{d}}^{2}=$ variance génétique additive de $\mathrm{P}_{d}$,

$\sigma_{1}^{2}=$ variance de l'indice moyen de la famille mesurée,

$i \quad=$ intensité de sélection.

Précisons que $\mathrm{E}\left(\Delta \mathrm{G}_{d}\right)_{\mathrm{A} \cdot \mathrm{B}}$ ne concerne que le progrès dû̀ à l'action des gènes additifs, de même que $\mathrm{E}_{(}\left(\Delta \mathrm{G}_{d}\right)_{\mathrm{c}}$; la différence entre les deux méthodes ne provenant alors, pour $i$ et $\sigma \bar{I}$ égaux, que de leurs caractéristiques propres : nombre d'individus par famille, sélection sur un sexe pour A.B, sur les 2 sexes pour C.

Par rapport à une sélection portant uniquement sur $\mathrm{P}_{s}$, l'utilisation de l'indice avec restriction multiplie l'expression du progrès génétique par $\mathrm{I}-r_{\mathrm{G}}^{2}$ dont la valeur est comprise entre o et $\mathrm{I}$.

\section{Résultats}

\section{I. - Expérience $I$}

Les principaux résultats sont rassemblés dans les tableaux 2 à 5 . Des analyses préliminaires (analyses de variance factorielles) dont nous ne détaillons pas ici les résultats ont montré l'absence de différences significatives entre les moyennes de la génération des mères et celles de la génération des filles pour chacune des six cellules période $\times$ milieu de ponte (ceci était d'ailleurs à prévoir en l'absence de toute sélection), ce qui nous a permis de faire des estimations globales pour les 2 générations et l'ensemble des répétitions (tabl. 2). Quelle que soit la période, la ponte en milieu dégrałé est très inférieure à celle en milieu standard (réduction moyenne fde 62 p. I00). En milieu standard (S-S), la période n'a pas d'influence significative sur le niveau de ponte; par contre,en milieu dégradé ( $D-D)$, la ponte est inférieure en période $2(\mathrm{P}<0, \mathrm{OOI})$. 


\section{TABLEAU 2}

Moyennes et variances de la ponte des femelles vierges pour les deux générations de l'expérience I Means and variances of egg laying of virgin females for the two generations of experiment I

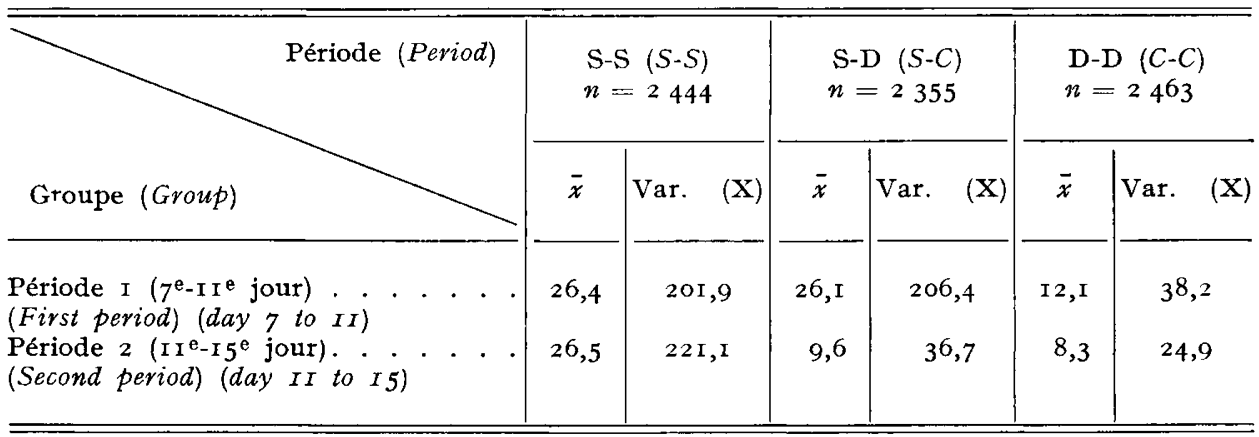

L'estimation de l'héritabilité calculée à partir du coefficient de régression $\beta$ de la moyenne des filles sur la ponte de la mère $\left(h_{\mathrm{R}}^{2}=2 \beta\right)$ est systématiquement inférieure à celle calculée à partir de la corrélation intra-classe $t$ par analyse de variance $\left(h_{\mathrm{s}}^{2}=2 t=\frac{2 \sigma_{\mathrm{F}}^{2}}{\sigma_{\mathrm{F}}^{2}+\sigma_{\mathrm{E}}^{2}}\right)($ tabl. 3 et 4$)$ quel que soit le groupe ou la période de ponte.

Pour chacune des méthodes d'estimations utilisées, l'héritabilité de la ponte n'est pas significativement différente pour un milieu donné entre les deux périodes ni pour une période donnée entre les deux milieux (tabl. 3).

\section{TABLEAU 3}

Expérience $I$ :

Estimations de l'héritabilité pour chaque cellule période de ponte $\times$ milieu de ponte par régression mère-fille $\left(h_{\mathrm{R}}^{2}\right)$ et par analyse de variance $\left(h_{\mathrm{g}}^{2}\right)$

Estimates of heritability for each cell period $\times$ environment of egg laying by daughter-dam regression $\left(h_{\mathbf{R}}^{2}\right)$ and by full sister analysis of variance $(h \mathbf{\mathrm { S }})$

\begin{tabular}{|c|c|c|c|c|c|c|}
\hline Groupe (Group) & \multicolumn{2}{|c|}{ S-S $(C-C)$} & \multicolumn{2}{|c|}{$\mathrm{S}-\mathrm{D} \quad(S-C)$} & \multicolumn{2}{|c|}{$\mathrm{D}-\mathrm{D}(C-C)$} \\
\hline Période (Poriod) & $h_{\mathrm{R}}^{2} \pm \sigma h_{\mathrm{R}}^{2}$ & $h_{\mathrm{S}}^{2} \pm \sigma h_{\mathrm{S}}^{2}$ & $h_{\mathrm{R}}^{2} \pm \sigma h_{\mathrm{R}}^{2}$ & $h_{\mathrm{g}}^{2} \pm \sigma h_{\mathrm{g}}^{2}$ & $h_{\mathbf{R}}^{2} \pm \sigma k_{\mathbf{R}}^{2}$ & $h_{\mathbb{S}}^{2} \pm \sigma h_{\mathbb{S}}^{2}$ \\
\hline $\begin{array}{l}\text { Période I }\left(7^{\mathrm{e}}-\mathrm{I} \mathrm{I}^{\mathrm{e}} \text { jour }\right) . \\
\text { (First period) }(\text { day } 7 \text { to } I I)\end{array}$ & $\begin{array}{r}0,20 \\
\pm 0,06\end{array}$ & $\begin{array}{r}0,39 \\
+0,05\end{array}$ & $\begin{array}{r}0,25 \\
\pm 0,06\end{array}$ & $\begin{array}{r}0,32 \\
\pm 0,06\end{array}$ & $\begin{array}{r}0,27 \\
\pm 0,05\end{array}$ & $\begin{array}{r}0,35 \\
\pm 0,05\end{array}$ \\
\hline $\begin{array}{l}\text { Période } 2 \text { (I } \mathrm{I}^{\mathrm{e}}-\mathrm{I} 5^{\mathrm{e}} \text { jour) } \\
\text { (Second period) (day II to I } 5)\end{array}$ & $\begin{array}{r}0,22 \\
\pm 0,05\end{array}$ & $\begin{array}{r}0,25 \\
\pm 0,05\end{array}$ & $\begin{array}{r}0,15 \\
\pm 0,05\end{array}$ & $\begin{array}{r}0,33 \\
\pm 0,06\end{array}$ & $\begin{array}{r}0,21 \\
\pm 0,04\end{array}$ & $\begin{array}{r}0,30 \\
\pm 0,05\end{array}$ \\
\hline
\end{tabular}


La corrélation phénotypique entre les deux périodes de ponte n'est pas modifiée par la nature du milieu (tabl. 5). La corrélation génétique $r_{\mathrm{G}}$ entre la ponte des deux périodes est très élevée dans les trois groupes. L'écart-type de $r_{\mathrm{G}}$ ne peut être valablement calculé que si cette corrélation est faible. On peut donc penser que l'estimation de $\sigma r_{\mathrm{G}}$ est biaisée.

TABLEAU 4

Estimations globales des héritabilités pour chaque milieu ou pour chaque période calculées par régression $\left(h_{\mathrm{R}}^{2}\right)$ et par analyse de variance $\left(h_{\mathrm{S}}^{2}\right)$ pour l'expérience $I$

Overall estimates of heritabilities for each environment or for each period calculated by regression $\left(h_{\mathbf{R}}^{2}\right)$ and by analysis of variance $\left(h_{\mathbf{S}}^{2}\right)$ for the experiment $I$

\begin{tabular}{|c|c|c|}
\hline & $h_{\mathbf{R}}^{2} \pm \sigma h_{\mathbf{R}}^{2}$ & $h_{\mathrm{S}}^{2} \pm \sigma h_{\mathrm{S}}^{2}$ \\
\hline $\begin{array}{l}\text { Ponte en milieu standard (quelle que soit la période) } \\
\text { (Egg laying in standard medium) (whatcver the period) }\end{array}$ & $0,22 \pm 0,04$ & $0,32 \pm 0,04$ \\
\hline $\begin{array}{l}\text { Ponte en milieu dégradé (quelle que soit la période) } \\
\text { (Egg laying in conditioned medium) (whatever the period) }\end{array}$ & $0,2 \mathrm{I} \pm 0,04$ & $0,33 \pm 0,04$ \\
\hline $\begin{array}{l}\text { Ponte du } 7^{\mathrm{c}} \text { au } \mathrm{I}_{\mathrm{I}}^{\mathrm{e}} \text { jour (quel que soit le milieu) } \\
\text { (Egg laying from day } 7 \text { to } I I \text { ) (whatcver the environment) }\end{array}$ & $0,24 \pm 0,04$ & $0,35 \pm 0,04$ \\
\hline $\begin{array}{l}\text { Ponte du I } \mathrm{e} \text { au } 5^{\mathrm{e}} \text { jour (quel que soit le milieu). } \\
\text { (Egg laying from day II to } 5 \text { ) (whatcver the cnvironment) }\end{array}$ & $0,19 \pm 0,04$ & $0,3^{\circ} \pm 0,04$ \\
\hline
\end{tabular}

\section{TABLEAU 5}

Estimations de la corvélation génétique $\left(r_{\mathrm{G}}\right)$ et phénotypique $\left(\gamma_{\mathbf{P}}\right)$ et de leur écart-type entre la ponte en ${ }_{I^{\mathrm{r}}}^{\mathrm{r}}$ et $2^{\mathrm{e}}$ période pour chacun des 3 groupes de l'expérience $I$

Estimates of genetic $\left(r_{\mathbf{G}}\right)$ and phenotypic $\left(r_{\mathbf{P}}\right)$ correlations and their standard error between egg laying in first and second period for each of the 3 groups of the experiment $I$

\begin{tabular}{|c|c|c|c|c|}
\hline & Groupe (Group) & S-S $(S-S)$ & $\mathrm{S}-\mathrm{D} \quad(S-C)$ & $\mathrm{D}-\mathrm{D}(C-C)$ \\
\hline $\begin{array}{l}\gamma_{G} \pm \sigma \gamma_{G} \\
r_{P} \pm \sigma \sigma_{P}\end{array}$ & 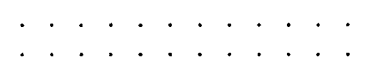 & $\begin{array}{l}\mathrm{I}, \mathrm{I} 4 \pm 0,05 \\
\mathrm{o}, 38 \pm 0,03\end{array}$ & $\begin{array}{l}0,87 \pm 0,03 \\
0,39 \pm 0,03\end{array}$ & $\begin{array}{l}0,72 \pm 0,06 \\
0,38 \pm 0,03\end{array}$ \\
\hline
\end{tabular}

\section{2. - Expérience $I I$}

Dans les tableaux 6 et 7 sont portés les résultats des deux types de sélection, pour chaque répétition, et sur les figures 3 à 5 l'évolution des moyennes de ponte (par répétition, et pour l'ensemble des deux) en fonction du numéro de génération. Le coefficient de régression $b$ de l'indice utilisé pour sélectionner était le même 
GÉNÉTIQUE DE LA PONTE CHEZ TRIBOLIUM

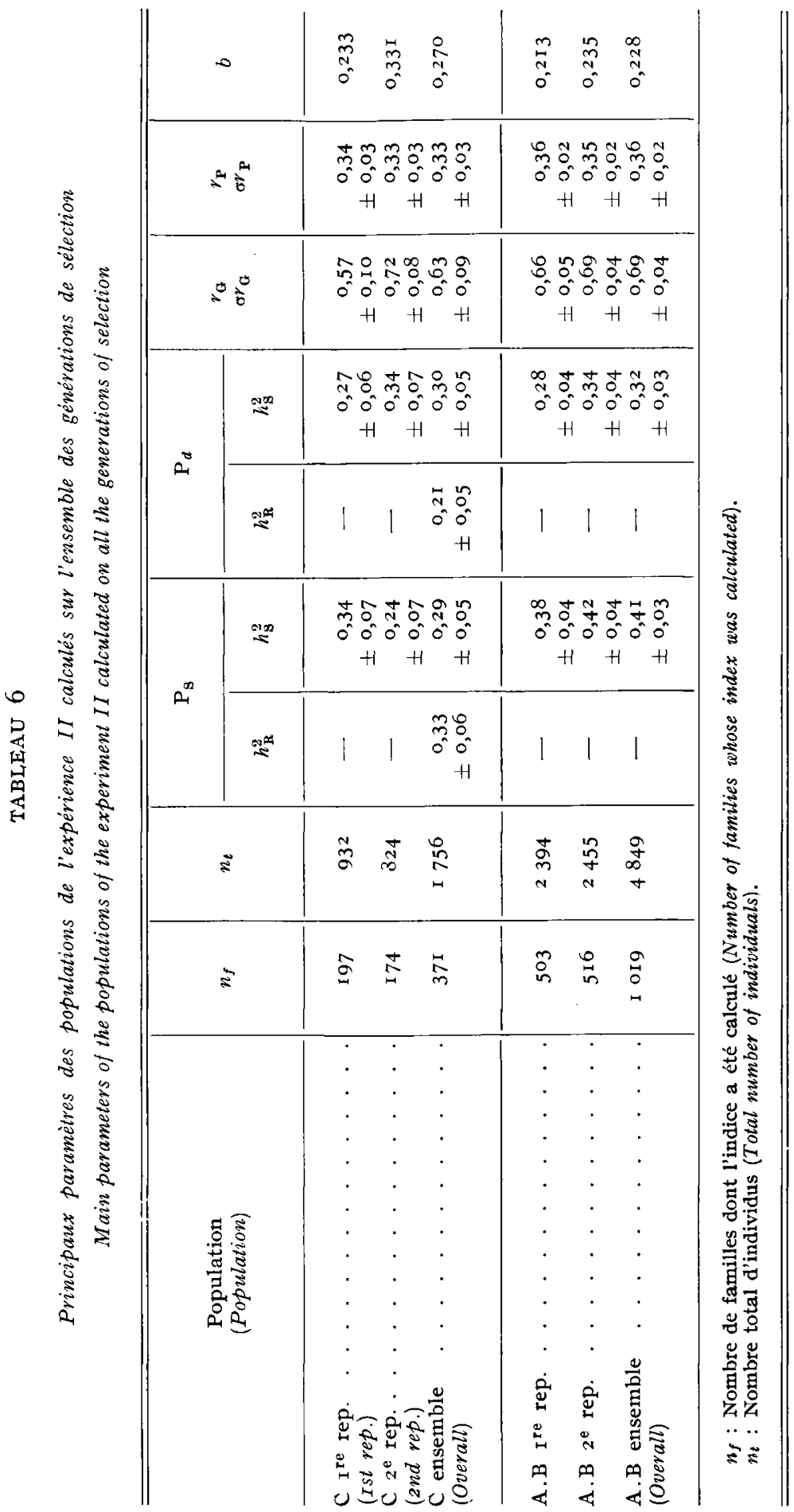


pour les deux types de sélection, et le même pour les deux répétitions pendant les trois premières générations. A partir de la $4^{\mathrm{e}}$ génération, le nombre de données était suffisant pour le calculer pour chaque répétition indépendamment. Dans le tableau 6 apparaissent les principaux paramètres calculés sur l'ensemble des générations de sélection. Pour la population $\mathrm{C}$, il a été possible de calculer l'héritabilité par corrélation entre pleines søurs (comme pour les populations $\mathrm{A} \times \mathrm{B}$ et $\mathrm{B} \times \mathrm{A}$ )

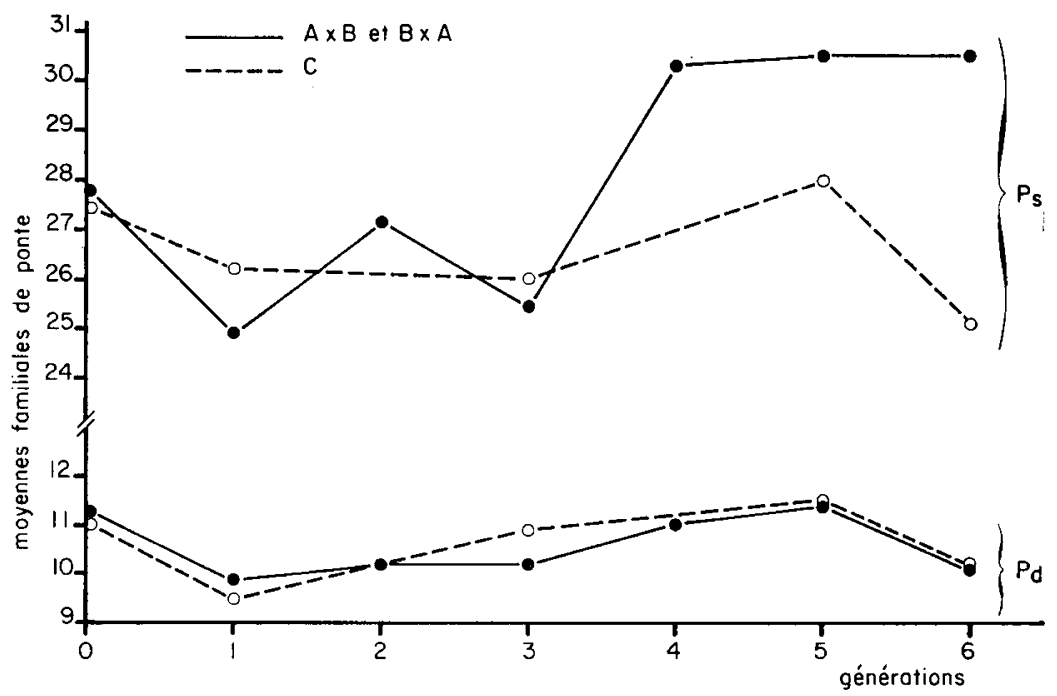

'IG. 3. - Évolution des moyennes de ponte en milieu standard $\left(P_{s}\right)$ et en milieu dégradé $\left(P_{d}\right)$ pour les deux répétitions de l'expérience II.

Variation of average egg laying in standard $\left(L_{s}\right)$ or conditioned $\left(L_{c}\right)$ medium for the two replicates of the experiment II.

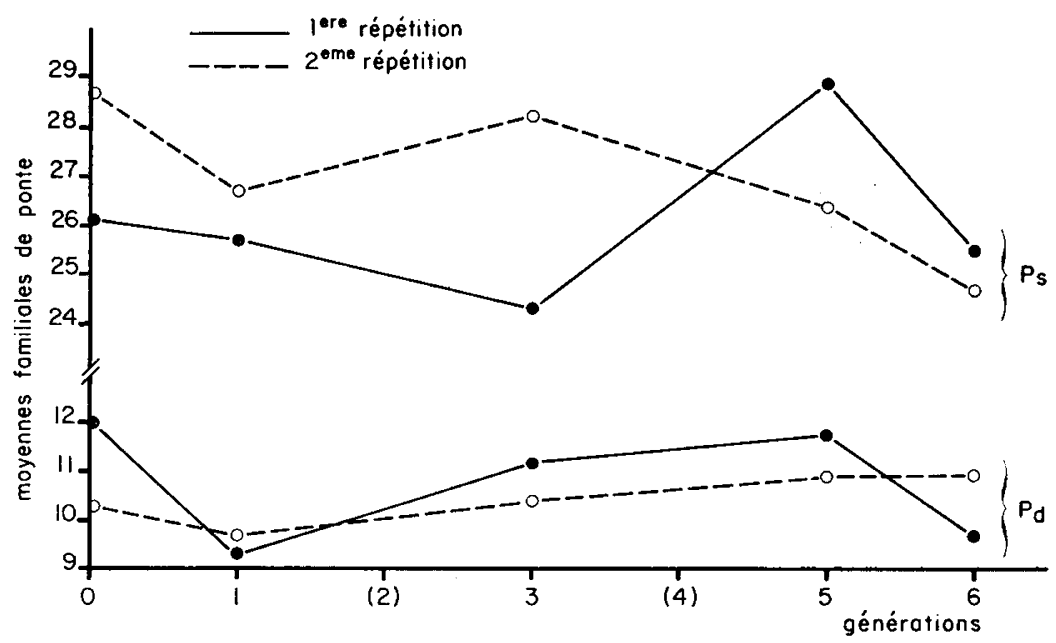

FIG. 4. - Expérience II. Moyennes de ponte des populations $C$ (sélection familiale). Experiment II. Average egg laying of populations $C$ (family selection). 


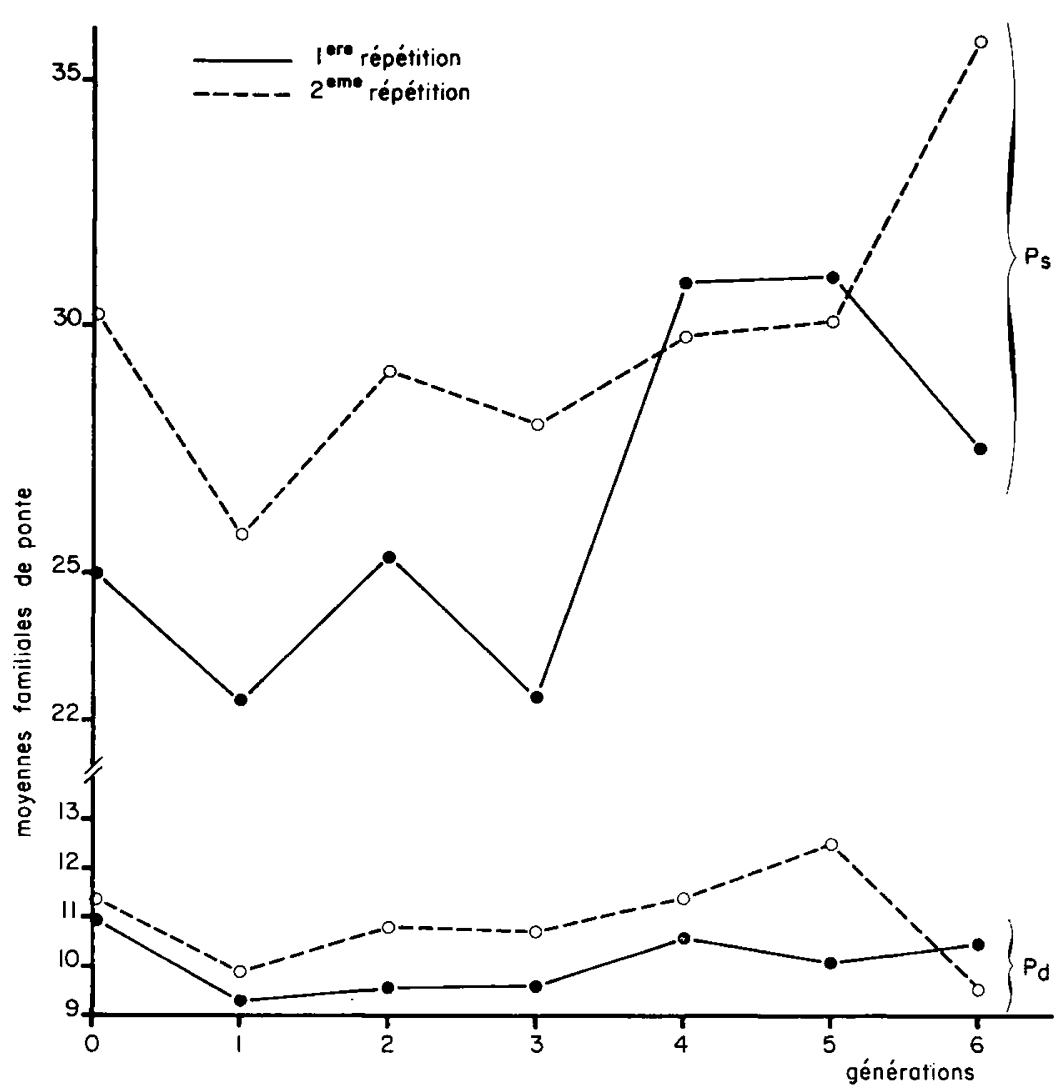

FIG. 5. - Expérience II. Moyennes de ponte des populations $A \times B$ et $B \times A$ (sélection réciproque récurvente).

Experiment II. Average egg laying of populations $A \times B$ and $B \times A$ (reciprocal recurrent selection).

mais aussi par régression de la moyenne des filles sur la performances de leur mère. Dans la population C, il n'y a pas de différence significative entre l'héritabilité de la ponte en milieu standard et celle de la ponte en milieu dégradé; par contre, dans les populations $\mathrm{A} \times \mathrm{B}$ et $\mathrm{B} \times \mathrm{A}$, 1'héritabilité de la ponte en milieu standard est très significativement supérieure $(P<0,05)$; 1'héritabilité calculée par régression des filles sur la mère est significativement inférieure à celle calculée par corrélation entre sœurs $(\mathrm{P}<0, \mathrm{IO})$ pour la ponte en milieu dégradé mais n'est pas significativement différente pour la ponte en milieu standard.

Rappelons que le but de la sélection était d'améliorer la ponte en milieu dégradé tout en la maintenant constante en milieu standard. Or, les seules évoluiions significatives des performances au cours de la sélection sont (fig. 3 à 5 , tabl. 7 ) :

- une augmentation de la ponte en milieu standard dans les populations $\mathrm{A} \times \mathrm{B}$ et $\mathrm{B} \times \mathrm{A}$ (sélection réciproque récurrente) $(\mathrm{P}<0,05)$,

- une faible augmentation de la ponte en milieu dégradé $(\mathrm{P}<0, \mathrm{ro})$ et une diminution de la ponte en milieu standard $(P<0,05)$ dans la deuxième répétition de la sélection familiale, mais aucune évolution nette pour l'ensemble des deux répétitions. 
Régression de la moyenne pour le caractère mesuré sur le numéro de génération Regression of the mean for the character on generation number

\begin{tabular}{|c|c|c|c|c|c|c|c|c|c|c|c|c|}
\hline & & & & & & & & & & \multicolumn{3}{|c|}{ Régression } \\
\hline & & & & & & & & & & $\mathrm{P}_{8}$ & & $\mathrm{P}_{a}$ \\
\hline 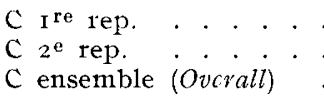 & $\begin{array}{l}\cdot \\
\cdot \\
\cdot\end{array}$ & $\begin{array}{ll}\cdot & \cdot \\
\cdot & \cdot \\
\cdot & \cdot\end{array}$ & $\begin{array}{l}. \\
.\end{array}$ & $\dot{\cdot}$ & $\dot{.}$ & $\dot{.}$ & . & $\dot{.}$ & $\cdot$ & $\begin{array}{l}0, \mathrm{I} 8 \pm 0,6 \mathrm{I} \\
-0,83 \pm 0,32^{* *} \\
-0,3 \mathrm{0} \pm 0,40\end{array}$ & $\begin{array}{r}-0,2 \mathrm{I} \\
0,24 \\
0,04\end{array}$ & $\begin{array}{l} \pm 0,42 \\
\pm 0,11^{*} \\
\pm 0,28\end{array}$ \\
\hline $\begin{array}{l}\text { A.B } \text { I }^{\text {re rep. }} . . \\
\text { A.B } 2^{\mathrm{e}} \text { rep. } \\
\text { A.B ensemble }(\text { Overall })\end{array}$ & . & $\begin{array}{ll}\cdot & \cdot \\
\cdot & \cdot \\
\cdot & \cdot\end{array}$ & . & $\dot{\cdot} \cdot$ & $\dot{.}$ & $\dot{.}$ & $\dot{r}$ & $\dot{.}$ & . & $\begin{array}{l}\mathrm{I}, 08 \pm 0,56^{*} \\
0,92 \pm 0,48^{*} \\
0,8 \mathrm{I} \pm 0,35^{* *}\end{array}$ & $\begin{array}{l}0,03 \\
0,001 \\
0,02\end{array}$ & $\begin{array}{l} \pm 0, \mathrm{I} 3 \\
\pm 0, \mathrm{I} 3 \\
\pm 0, \mathrm{I} 3\end{array}$ \\
\hline
\end{tabular}

\section{Discussion}

\section{1. - Expérience I}

Nous avons estimé l'héritabilité de la ponte des femelles vierges de Tribolium par 2 méthodes : à partir du coefficient de régression " $\beta$ » de la performance des filles sur la performance de leur mère $\left(h_{\mathrm{R}}^{2}=2 \beta\right)$ et à partir de la corrélation génétique " $t$ " entre les performances des pleines sœurs $\left(h_{\mathrm{s}}^{2}=2 t\right)$.

Pour une même méthode de calcul, l'absence de différences significatives entre les héritabilités indique que la part de la variance génétique additive est la même quelle que soit la période $\left(7^{\mathrm{e}_{-I} \mathrm{I}^{\mathrm{e}}}\right.$ ou II $\mathrm{I}_{-I} 5^{\mathrm{e}}$ jour $)$ et quel que soit le milieu (standard ou dégradé) (tab1. 4). Par contre il apparaît une différence très significative $(\mathrm{P}<0,05)$ entre $h_{\mathrm{R}}^{2}$ et $h_{\mathrm{s}}^{2}$, d'une valeur moyenne de $0, \mathrm{II}$. Elle provient d'effets génétiques non additifs et/ou d'effets de milieu commun.

Si l'on écarte l'hypothèse de la présence d'effets maternels d'origine génétique et la présence d'effets d'épistasie, on obtient les relations suivantes :

$$
\begin{gathered}
h_{\mathrm{R}}^{2}=\frac{\mathrm{V}_{\mathrm{A}}}{\mathrm{V}_{\mathrm{P}}} \\
\left.h_{\mathrm{s}}^{2}=\frac{\mathrm{V}_{\mathrm{A}}}{\mathrm{V}_{\mathrm{P}}}+\frac{\mathrm{I}}{2} \frac{\mathrm{V}_{\mathrm{D}}}{\mathrm{V}_{\mathrm{P}}}+2 \frac{\mathrm{V}_{\mathrm{C}}}{\mathrm{V}_{\mathrm{P}}} \quad \text { (Falconer, } \mathrm{I} 960\right)
\end{gathered}
$$

où $V_{A}$ est la variance génétique additive,

$V_{D}$ la variance génétique de dominance,

$\mathrm{V}_{\mathrm{c}}$ la variance due à l'effet milieu commun.

$$
h_{\mathrm{g}}^{2}-h_{\mathrm{R}}^{2}=\frac{\mathrm{r}}{2} \frac{\mathrm{V}_{\mathrm{D}}}{\mathrm{V}_{\mathrm{P}}}+2 \frac{\mathrm{V}_{\mathrm{C}}}{\mathrm{V}_{\mathrm{P}}}=0, \mathrm{II} \pm 0,03 \quad \text { (écart-type approximatif). }
$$


Les valeurs maximales que peuvent prendre ces effets sont donc :

$\mathrm{V}_{\mathbf{D}}=22$ p. Ioo de la variance phénotypique en l'absence d'effet milieu commun, $\mathrm{V}_{\mathrm{c}}=5,5 \mathrm{p}$. roo de la variance phénotypique en 1'absence d'effets génétiques de dominance.

Les pleines sœurs restent dans le même milieu jusqu'au début de 1'expérience ( $7^{\mathrm{e}}$ jour du stade adulte); il semble donc que la présence d'un effet milieu commun représentant $5,5 \mathrm{p}$. roo de la variance phénotypique soit tout à fait possible. Par contre, la présence d'effets génétiques non additifs représentant $22 \mathrm{p}$. Ioo de la variance phénotypique en milieu standard serait en contradiction avec les études précédentes (OrOzco et BEL,, I974a et $b$; LOPEZ-FANJUL, et JODAR, I977).

En ce qui concerne la ponte en milieu dégradé, aucune étude bibliographique ne nous permet d'écarter l'hypothèse d'une variance génétique non additive provenant soit d'un changement du mode d'action de gènes présents aux loci contrôlant la ponte en milieu standard, soit de 1' "activation" d'autres loci à action non additive. Dans la première hypothèse, le nombre de gènes agissant additivement est modifié, donc la part de la variance génétique additive devrait être différente dans les deux milieux, ce qui est contredit par nos estimations de l'héritabilité, calculées à partir de la régression; par contre, la deuxième hypothèse ne peut pas être rejetée formellement, bien qu'elle soit peu vraisemblable étant donné que les estimations de la corrélation génétique entre $\mathrm{P}_{s}$ et $\mathrm{P}_{d}$ sont proches de $\mathrm{I}$.

Enfin, la présence d'un effet milieu commun est également possible. A ces questions, les résultats de l'expérience II peuvent apporter des éléments de réponse.

\section{2. - Expérience $I I$}

\section{Estimation des paramètres génétiques}

Bien que ce ne soit pas le but de cette expérience, nous pouvons observer les valeurs obtenues pour les héritabilités et la corrélation génétique. Chez les animaux soumis à la sélection familiale (population C) nous avons pu calculer l'héritabilité par les deux mêmes méthodes que dans l'expérience $\mathrm{I}$, alors que chez ceux soumis à la sélection réciproque récurrente (populations $\mathrm{A}$ et $\mathrm{B}$ ), nous ne 1'avons calculée que par analyse de variance $\left(h_{\S}^{2}\right)$; 1'héritabilité de la ponte en milieu standard est significativement supérieure à celle en milieu dégradé quand elle est calculée par régression dans la population $\mathrm{C}(\mathrm{P}<0, \mathrm{IO})$ et par analyse de variance dans les populations $\mathrm{A} \times \mathrm{B}$ et $\mathrm{B} \times \mathrm{A}(\mathrm{P}<0,05)$. Dans la population $\mathrm{C}$, il n'y a pas de différence significative entre les deux méthodes de calcul pour $\mathrm{P}_{s}$, mais on retrouve les résultats de l'expérience A pour $\mathrm{P}_{d}\left(h_{\mathrm{s}}^{2}=0,09 \pm 0,07\right)$ bien que moins nettement.

\section{Résultats de la sélection}

Dans nos conditions théoriques $\left(i=\mathrm{I}, 37, \sigma_{\mathrm{G}_{S}}^{2} \simeq \mathrm{I} 0, \sigma_{\overline{\mathrm{I}}} \simeq 0,05\right)$ on peut calculer l'espérance du progrès génétique à partir des formules (I) et (2) :

$\mathrm{E}\left(\Delta \mathrm{G}_{s}\right)_{\mathrm{C}}=\mathrm{I}, 27$ œuf/génération $=0,38$ unité d'écart-type.

$\mathrm{E}\left(\Delta \mathrm{G}_{\delta}\right)_{\mathrm{A} \cdot \mathbf{B}}=0,5^{8}$ œuf/génération $=0, \mathrm{I} 7$ unité d'écart-type.

Comme la valeur de la corrélation génétique est de l'ordre de 0,70 on voit que l'espérance du progrès génétique sur $\mathrm{P}_{d}$ est divisée par 2 par rapport à un indice de sélection sans restriction $\left(I-r_{G}^{2} \simeq 0,5^{\circ}\right)$. 
En fait, l'intensité de sélection théorique a été impossible à respecter, en raison de la mortalité des animaux destinés à donner la génération suivante et des femelles contrôlées; on peut considérer que l'intensité de sélection a été réduite de 20 à 30 p. IOo.

L'index de sélection avec restriction a été utilisé avec succès par un certain nombre d'auteurs (OKADA et HARDIN, I967-I970; SCHEINBERG et al., I967; ABPI $A$ ANALP et al., I963). Cependant, on peut noter que en général le progrès réalisé est inférieur au progrès attendu. D'autre part, la précision de la prévision du progrès génétique dépend de la précision de l'estimation des paramètres; or nous obtenons des valeurs élevées pour le coefficient de corrélation génétique et nous savons que l'estimation du coefficient de corrélation est d'autant moins précise que sa valeur est élevée. On peut s'attendre à ce que le progrès génétique soit d'autant plus faible que cette corrélation génétique est élevée. Cependant $\mathrm{Mc}$ CARThy et DoolitTLE (I977), avec deux caractères très hautement corrélés, obtiennent une réponse significative sur le caractère non fixé. MALLARD (I972), tout en montrant que 1'utilisation de cet index peut être étendue à des cas de restrictions plus complexes, souligne la grande variabilité des résultats obtenus.

\section{- En ce qui concerne la sélection familiale (population C) :}

Pour la ponte en milieu standard, la réponse à la sélection a été nulle comme on pouvait s'y attendre puisque c'était précisément le but de l'indice avec rectriction utilisé. Puisque la réponse en milieu dégradé a été nulle également, on peut en déduire que la corrélation génétique $\gamma_{G}$ entre la ponte dans les deux milieux doit être voisine de I (donc $I-r_{\mathrm{G}}^{2}$ est nul). Ce résultat concorde bien avec les estimations de $r_{G}$ obtenues dans l'expérience I et dans cette même expérience. On peut donc conclure que, en ce qui concerne les effets additifs, ce sont les mêmes gènes avec les mêmes effets qui contrôlent le caractère dans les deux milieux. En d'autres termes on peut apporter une réponse à une des questions soulevées par 1'expárience A, c'est-à-dire qu'il n'existe pas d'effets additifs des gènes contrôlant spécifiqu?ment la résistance aux conditions difficiles.

\section{- En ce qui concerne la sélection réciproque récurrente (populations $\mathrm{A}$ et $\mathrm{B}$ )}

Rappelons tout d'abord que la théorie des indices de sélection, de même que la prédiction de la réponse à la sélection, ne sont basées que sur l'effet additif des gènes. Aussi ne pouvions-nous pas prédire l'importance de l'éventuelle action génique non additive que la sélection réciproque récurrente pouvait mettre en évidence. Quoi qu'il en soit, il est indiscutable que notre hypothèse de travail, qui supposait un effet de surdominance pour la ponte en milieu dégradé, ne s'est pas vérifiée; au contraire, l'augmentation de la ponte en milieu standard, légère mais significative, peut être due à une action non additive des gènes en milieu standard. Donc, si 1'hypothèse d'identité dans les deux milieux des gènes additifs se trouve confirmée, nous n'avons pas pu vérifier l'existence d'une action de type non additif différente suivant le milieu; si elle existe, elle ne peut qu'être peu importante. La seul argument en sa faveur serait la différence significative d'héritabilité entre $\mathrm{P}_{s}$ et $\mathrm{P}_{d}$ dans la population $\mathrm{C}$, résultat qui peut se recouper avec une des conclusions de l'expérience I (où̀ l'on ne pouvait pas trancher entre effet de dominance et effet milieu commun) mais n'est étayé par aucun des autres résultats.

Enfin, il convient de faire une remarque concernant l'efficacité et la rapidité de la réponse avec la procédure utilisée pour ce type de sélection : en effet, le système utilisé est peut-être le plus intéressant pour étudier la surdominance, mais il présente un handicap important au départ, puisque l'on part d'une population 
non sélectionnée que 1'on divise en deux au hasard à la première génération, ce qui fait que les fréquences des allèles aux éventuels loci avec surdominance sont pratiquement égales au départ; or, l'on sait que la sélection réciproque récurrente est d'autant plus efficace que l'on utilise deux populations hautement sélectionnées en directions opposées (c'est-à-dire avec des fréquences des allèles non additifs très différentes) (Chevale't et Gillois, I976).

\section{Conclusion}

Des deux étapes de cette étude, la première de type statique, la suivante de type dynamique, on peut tirer les conclusions suivantes :

- il n'existe pas de gènes à effet additif différents de ceux agissant en milieu normal, qui contrôlent la ponte en milieu défavorable;

- contrairement aux résultats obtenus chez Tribolium dans d'autres types de milieux défavorables (la température ambiante) par OROzco et BELL (I974a et $b$ ), nous n'avons pas pu mettre en évidence l'action de gènes agissant de façon non additive pour contrôler la résistance à un milieu défavorable. Cependant, si ces gènes existent les résultats de l'expérience de type statique ont montré que leur action n'explique qu'une part peu importante de la variance génétique. Pour apporter de nouveaux éléments de réponse, il faudrait réaliser une expérience permettant de mesurer 1'importance de l'effet milieu commun chez les femelles vierges. On peut également se demander si l'apparente complexité des phénomènes de régulation de la ponte en fonction du milieu alimentaire (SokoLoFF, I974) ne retire pas beaucoup d'intérêt à ce caractère comme modèle simple d'étude des mécanismes de l'interaction génotype $\times$ milieu.

Reçu pour publication en décembre 1979.

\section{Remerciements}

Nous tenons à remercier R. DIEz BARRA, du Département de Génétique de l'I.N.I.A. à Madrid, pour la réalisation des programmes sur ordinateur.

\section{Summary}

The genetic determinism of egg laying in virgin females of Tribolium castaneum in standard or conditioned medium

With the aim of studying the genetic determinism of the resistance to unfavourable environmental conditions and in particular to verify the hypothesis according to which it is mainly depending on the action of non additive genes, we used the trait " egg laying of virgin females of Tribolium Castaneum "from day 7 to I I (first period) and from day I I to I 5 (second period) of adult life. The animals were kept either in a standard medium (S) or in a highly conditioned medium (C). The first experiment involving three groups of six hundred females plus three daughters of each was made in the following media, S-S, S-C, C-C (first and second period). The 
egg laying in the conditioned medium was $60 \mathrm{p}$. Ioo lower than in the stardard medium. The heritability was not significantly affected by the egg laying period or environment. Conversely, the heritability calculated by the correlation between the three full sibs (o.33) significantly exceeded that calculated by daughter-dam regression (0.22). This might be due to non additive genetic effects and /or to common environmental effect in the daughters.

In the second experiment, we selected the animals using an index with restriction to increase the egg laying in conditioned medium $\left(\mathrm{L}_{c}\right)$, in the second period, without modifying that of the stardard medium $\left(\mathrm{L}_{s}\right)$, in the first period. As foreseen, the family selection did not affect either $\mathrm{L}_{s}$ or $\mathrm{L}_{c}$ whereas the reciprocal recurrent selection, contrary to our hypothesis, had no influence on $\mathrm{L}_{c}$ ard increased $\mathrm{L}_{i s}$.

It may be concluded from these experiments on the one hand that the aciditive genes are the same in standard or conditioned media and on the other that it is impossible to draw a definitive conclusion on the existence of resistance genes acting by dominance.

\section{Références bibliographiques}

Abplanaly H., OGaswara F. X., Asmundson V. S., 1963. Influence of selection for body weight at different ages in turkeys. Brit. Poult. Sci., 4, 71-82.

BARNETT S. A., Coleman Elisabeth M., 1960. "Heterosis" in $F_{1}$ mice in a cold envircnment. Genet. Res., 1, 25-38.

Chevalet C., Gilrois M., I976. L'hétérosis. Aspects théoriques et expérimentavx. In Bull. tech. Dép. Génét. anim. ('Inst. nat. Rech. agron., Fr.), no 24, 5-22.

Falconitr D. S., ig60. Introduction to quantitative genetics. Oliver and Boyd, Edimburgh. KEMPTHORNE O., NORDSKOG A. W., I959. Restricted selection indices. Biometrics, $15,10-19$.

LAVIE B., RitTe U., MoAv R., I978. The genetic basis of egg lay response to conditioned medium in the flour beetle, Tribolium Castaneum. I. Two way selection. Theor. Appl. Genct., 52, I93-I99.

LOPEZ-FANJUL C., JODAR B., I977. The genetic properties of egg laying of virgin females of Tribolium Castaneum. Heredity, 39, $25^{\mathrm{I}-258 .}$

MaLlakd J., I972. La théorie et le calcul des index de sélecticn avec restrictions : synthèse critique. Biometrics, 28, 7I 3-735.

Mc Carthy J. C., Doolititle D. P., I977. Effects of selection for independent changes in two highly correlated body weight traits of mice. Genet. Res. Camb., 29, I 33-I 45.

OKADA I., HARDIN R. T., 1967. An experimental examination of restricted selection index, using Tribolium Castaneum. I. The results of two way selection. Gcnctics, 57, 227-236.

OKADA I., HaRdin R. T., I970. An experimental examination of restricted selection index, using Tribolium Castaneum. II. The results of long term one way selection. Genetics, 64, 533539 .

Orozco F., I976. A dynamic study of genotype-environment interaction with egg laying of Tribolium castaneum. Hevedity, 37, I57-I 71.

Orozco F., BELL A. E., I974a. A genetic study of egg laying of Tribolium in optimal and stress environments. Can. J. Genet. Cytol., 16, 49-6o.

Orozco F., BELI, A. E., I $974 b$. Reciprocal recurrent selection compared to within strain selection for increasing rate of egg lay of Tribolium under optimal and stress conditions. Genetics, 77, I 43-I6I.

Parsons P. A., I97I. Extreme environment heterosis and genetic loads. Hevedity, 26, $479-483$.

Scheinberg E., BELL A. E., ANDERson V. L., I967. Genetic gain in populations of Tribolium Castaneum under uni-stage tandem selection and under restricted select:on indices. Gcnetics, 55, 69-9o.

Sokolor A., I974. The biology of Tribolium, vol. 2, Oxford University Press, Oxford. 